\section{Fully equipped}

If patients are motivated enough to clean their teeth for two minutes, twice a day, they're going to want to be rewarded with good oral health, particularly good gingival health. As well as the right technique, they also need to be using the right products. Just as you want your washing machine and detergent to work in harmony, so too do you want a toothbrush and toothpaste to give the best possible outcome.

Oral-B’s Genius toothbrush works in synergy with Oral-B's Pro-Expert toothpaste. The Genius brush will almost certainly improve a patient's technique ensuring the user brushes for the right length of time, does not apply too much pressure and, most importantly, never misses a zone! There is no excuse for non-uniform brushing as real-time guidance is given to improve their technique.

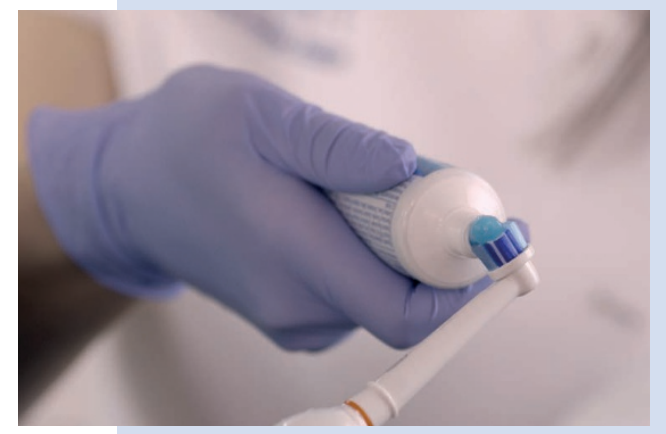

Using the washing machine analogy, Oral-B’s Pro-Expert is the 'Ariel' to its Genius power toothbrush. It's the inclusion of stabilised stannous fluoride that makes the difference. This powerful ingredient gives Oral-B's Pro-Expert toothpaste a long-lasting antimicrobial action as it inhibits antimicrobial growth as well as reducing the ability of bacteria to 'stick' to tooth and gum surfaces.

The inclusion of sodium hexametaphospate is also beneficial as it protects against calculus formation, as well as staining, thereby reducing a further cause of plaque retention on the roughened surface of the calculus. The combined force of these two plaque reducing agents, alongside the mechanical action of the Oral-B Genius help protect against gum problems.

\title{
New elevator with curved blade
}

Directa $A B$ is proud to announce that the NEW Luxator Forte F32C Elevator is now available in the United Kingdom.

The Luxator Forte Elevator differs from Luxator periotomes with its black handle, making it easily recognisable.

The stainless steel blade is thicker and has a slightly different design of the tip, allowing it to be used for elevation of the tooth, after cutting of the periodontal ligaments with a Luxator Periotome has been performed. The world acclaimed, ergonomic handle of the

Luxator instruments stays the same. The development of the Luxator Forte F32C initiated from dentists' desire to use a slightly curved instrument for their elevation. The curved blade will increase choice and improve accessibility for controlled and safer tooth elevation.

Contact your local dealer for more information and to order Luxator Forte F32C. Find more product information at: http://www.directadental. com/products/Luxator/ Luxator-forte-elevator.

\section{Automated production of precision restorations}

Roland DG Corporation has released its first dental 3D printer, the DWP-80S, to assist in the production of dentures. Launched simultaneously, the DWX-52DC is the newest addition to the DWX series dental mills and includes several new automated functions for the unattended production of precision dental restorations. Roland DG now offers the dental industry both additive (3D printing) and subtractive (milling) manufacturing processes to improve the workflow of dental technicians.

Since their launch in 2010, Roland DG's DWX series dental mills have gained industry recognition for their desktop size, user-friendliness, robust reliability and open architecture, which has contributed to the widespread digitalisation of dental restorations such as crowns and bridges. The DWX-52DC adds exciting new features to further automate and expand the formerly labour-intensive process.

With a newly developed Automatic Disc Changer (ADC) capable of storing up to six discs, the DWX-52DC is fully equipped to mill a variety of precision dental restorations overnight to increase lab productivity. The included pin-type material adapter enables the setting up and milling of seven pin-type blocks consecutively. With the DWX-52DC's expanded production capacity, a variety of dental restorations can be produced. The 15-station Automatic Tool Changer (ATC) automatically replaces milling burs without interruption for the efficient milling of a wide range of materials.

The DWP-80S uses a proprietary projector lens to cure resin materials with UV-LED light. Bundled with the DWP-80S, new Quick Denta software provides a remarkably simple solution for the $3 \mathrm{D}$ printing of 